\section{Horizon's X files}

\author{
By Kai-Jye Lou, Staff Writer
}

Italian researchers have developed cancer cell lines that they believe recapitulate in vivo conditions better than conventional recombinant cell lines. Horizon Discovery Ltd. is commercializing the cell lines, called $\mathrm{X}$-MAN, and already has 12 deals in place for the technology.

Researchers at the University of Turin Medical School have used a viral-based gene engineering platform to knock in tumor-associated mutations in normal human somatic cells. ${ }^{1}$ The resulting mutant cell lines, in combination with their unmutated isogenic counterparts, provide a controlled cellular model system for patient profiling and drug screening studies.

"These cell lines can shorten the development timeline for products," said Alberto Bardelli, a group leader at the Institute for Cancer Research
"The idea is that if you have these paired cell lines, you can design targeted therapies because you have the ideal paired control."

-Ben Ho Park,

The Johns Hopkins University the cells' responses to drugs.

When evaluated against a panel of 98 antiproliferative compounds, several knock-in mutants displayed drug sensitivity and resistance profiles resembling those of naturally occurring tumor cells.

"The benefit of being able to use the rAAV technique to build cancer cells from the ground up promises to deliver a next generation of disease models that for the first time will challenge a potential lead compound with a series of relevant biological questions prior to the design and implementation of expensive clinical trials," Bardelli told SciBX.

\section{$\mathrm{X}-\mathrm{MAN}$ for the job}

According to Disley, X-MAN models enable "identification of novel cancer drugs that are optimally targeted to specific patient populations; prediction and selection of genetically relevant patients for shorter, more focused and cheaper clinical trials; profiling of rational drug combinations to develop new therapies that overcome mutation-driven drug resistance; and identification of biomarkers related to disease-causing mutations or polymorphisms that predict disease onset, prognosis and drug responsiveness."

He added that "by screening potential drug candidates on well-defined diseased vs. normal cell lines, clear information on a drug's mechaand Treatment (IRCC) and associate professor in the Department of Oncological Sciences at the University of Turin Medical School. He is a cofounder of Horizon Discovery and principal investigator on the study describing the cell lines, which was published in the Proceedings of the National Academy of Sciences.

In human cancers, malignant transformation in normal cells typically occurs after the accumulation of multiple genetic mutations. Expression of these mutated genes in the cell is controlled by the gene's native promoter region and regulatory pathways.

By contrast, many cellular models used to study cancer-associated genes involve modifying cells to express a mutated gene of interest. The problem is that the mutated genes are not under endogenous control. Instead, expression is typically controlled by a non-native viral promoter. In addition, these ectopic expression models typically involve only a single mutated target gene that is overexpressed at levels beyond those observed in human cancer cells. The result is a high incidence of false positives in compound screens.

"Ectopic gene expression systems fail to mimic complex regulation and feedback loops," said Darrin Disley, commercial director and chairman of Horizon Discovery. He added that because use of such models often results in false positives, they always require follow-up testing of the compound with large numbers of secondary assays.

Bardelli's group used recombinant adeno-associated virus (rAAV) vectors to knock in tumor-associated mutations in normal human somatic cells. Compared with ectopic expression models, these cells kept the mutated genes under endogenous control, which more accurately reproduces both the way in which malignant transformation occurs and nism, therapeutic window and responsiveness can now be obtained prior to entering expensive clinical trials."

In addition, Disley said, the underlying rAAV-based technology, called Genesis, allows for the creation of new cell line pairs in 3-6 months compared with up to 24 months for conventional DNA-based techniques.

Ben Ho Park, an associate professor of oncology at The Johns Hopkins University, said there are two main advantages of the paired X-MAN isogenic cell lines. First, he said, the cell lines are genetically stable, which is an improvement over established human cancer cell lines such as HCT116 and MCF-7.

Second, he said the background genetic differences between various human cancer cell lines are controlled for with the unmutated isogenic counterpart.

"The idea is that if you have these paired cell lines, you can design targeted therapies because you have the ideal paired control," Park told SciBX

Preclinical cancer company Agios Pharmaceuticals Inc. is using $\mathrm{X}$-MAN cell lines to "help us to identify representative cancer genotypes that present the best response to our drug, in hopes that it will not only generate IP for us, but also help in patient stratification in clinical trials," said Shin-San Michael Su, VP of drug discovery.

Moreover, he said, "we don't need to screen hundreds of cancer cell lines, animal models and clinical specimens with diverse genome types to come to a clear understanding as to what genetic background will most likely benefit from the class of drugs we are developing."

According to Markus Warmuth, executive director of oncology 
research at Novartis AG's Novartis Institutes for BioMedical Research, "what's particularly appealing about the X-MAN cell lines is that you can delete or change the genes" and preserve endogenous control.

He noted that conventional methods of creating cellular cancer models involve cDNA overexpression, which can cause cells to die or undergo cell cycle arrest. This can be misinterpreted as a positive result in drug screening assays and makes it challenging to establish a cell culture.

Su did note the X-MAN lines are not going to replace in vivo studies.

"The lack of a tumor microenvironment, tumor-host interaction and the lack of the whole battery of other factors, such as immune cells and their responses, might make it hard for X-MAN cell lines to recapitulate the various human cancer genotypes in real life," he said. "The major caveat regarding the use of cell lines is fundamentally unchanged-these are still cell lines and do not and cannot replace experimental systems in animals."

He added that each cell line only represents a single cell type, which "might not translate well into in vivo tumors, which are highly heterogeneous."

Going forward, Bardelli says his group is validating the observed sensitivity and resistance profiles detailed in the PNAS paper across multiple cellular models and working to generate additional lines carrying multiple mutations.
Use of X-MAN cell lines can be licensed from Horizon Discovery. The University of Turin has filed a patent application covering the generation of panels of X-MAN cell lines with knock-in mutations and their use in drug screening, which has been exclusively licensed to the biotech company.

Horizon also holds exclusive worldwide rights from the University of Washington to patents covering the Genesis rAAV technology. The biotech, in turn, licensed Genesis to the Department of Oncological Sciences at the University of Turin, which is using the IP to engineer cell lines for Horizon.

Lou, K.-J. SciBX 2(3); doi:10.1038/scibx.2009.81

Published online Jan. 22, 2009

\section{REFERENCES}

1. Di Nicolantonio, F. et al. Proc. Natl. Acad. Sci. USA; published online Dec. 8, 2008; doi:10.1073/pnas.0808757105

Contact: Alberto Bardelli, University of Turin Medical School, Turin, Italy

e-mail: a.bardelli@unito.it

\section{COMPANIES AND INSTITUTIONS MENTIONED}

Agios Pharmaceuticals Inc., Cambridge, Mass. Horizon Discovery Ltd., Cambridge, U.K.

The Johns Hopkins University, Baltimore, Md. Novartis AG (NYSE:NVS; SWX:NOVN), Basel, Switzerland University of Turin Medical School, Turin, Italy University of Washington, Seattle, Wash. 